\title{
Colonization and Infection with Antibiotic-Resistant Bacteria in a Long-Term Care Facility
}

\author{
Margaret S. Terpenning, ${ }^{* \neq}$ Suzanne F. Bradley, ${ }^{* \neq}$ Jim Y. Wan, ${ }^{\mathfrak{S}}$ Carol E. Chenoweth, ${ }^{\dagger \neq}$ \\ Karen A. Jorgensen," and Carol A. Kauffman ${ }^{\dagger \neq \|}$
}

OBJECTIVE: To assess colonization and infection with methicillin-resistant Staphylococcus aureus (MRSA), highlevel gentamicin-resistant enterococci (R-ENT) and gentamicin and/or ceftriaxone-resistant Gram-negative bacilli (R$\mathrm{GNB}$ ) and the factors that are associated with colonization and infection with these organisms.

DESIGN: Monthly surveillance for colonization and infection over a period of 2 years. In the second year, an intervention to decrease MRSA colonization by the use of mupirocin ointment was carried out.

SETTING: Long-term care facility attached to an acute care Veterans Affairs Medical Center.

PATIENTS: A total of 551 patients in the facility were followed for a period of 2 years.

MEASUREMENTS: Colonization and infection rates with MRSA, R-ENT, and R-GNB. Analysis of risk factors associated with colonization and infection with these three groups of organisms.

MAIN RESULTS: In the first year, colonization rates were highest for MRSA $(22.7 \pm 1 \%$ patients colonized each month) and R-ENT $(20.2 \pm 1 \%)$ and lower for R-GNB $(12.6 \pm 1 \%)$. After introduction of decolonization of nares and wounds with mupirocin, the rate of MRSA colonization fell significantly to $11.5 \pm 1.8 \%$, but rates remained unchanged for R-ENT and R-GNB. Risk factors for MRSA colonization included the presence of wounds and decubitus ulcers. For R-ENT, the presence of wounds, renal failure, intermittent urethral catheterization, low serum albumin, and poor functional level were significant. For R-GNB, intermittent urethral catheterization, chronic renal disease, inflammatory bowel disease, presence of wounds, and prior pneumonia were significantly associated with colonization. Overall, of infections caused by known organisms, $49.6 \%$ were due to MRSA, R-ENT, or R-GNB, and $50.4 \%$ were

From the Divisions of "Geriatric Medicine and IInfectious Diseases, $¥$ Department of Internal Medicine, The University of Michigan Medical School; SDepartment of Biostatistics, University of Michigan School of Public Health; and IDepartment of Veterans Affairs Medical Center, Ann Arbor, Michigan. Dr. Wan is now with the Division of Biostatistics and Epidemiology, University of Tennessee, Memphis, Tennessee.

This work was supported by the Veterans Affairs Health Services Research and Development Service.

Address correspondence and reprint requests to Dr. Margaret S. Terpenning, GRECC 11G, Veterans Affairs Medical Center, 2215 Fuller Road, Ann Arbor, MI 48105. due to susceptible organisms. Infections were more commonly due to R-GNB (21.1\% of all infections) than to R-ENT $(8.3 \%)$ or MRSA (4.6\%). The most common infections were urinary tract infections $(42.9 \%$ of all infections) and skin and soft tissue infections (31.9\% of all infections). Risk factors for MRSA infections were diabetes mellitus and peripheral vascular disease, for R-GNB infections were intermittent urethral catheterization and indwelling urethral catheters, and no one factor was associated with R-ENT infection.

CONCLUSIONS: In our long-term care facility, colonization with resistant MRSA and R-ENT was more common than R-GNB, but infections were more often due to R-GNB than R-ENT and MRSA. Several host factors, which potentially could be modified in order to prevent infections, emerged as important in colonization and infection with these antibiotic-resistant organisms. J Am Geriatr Soc 42:1062-1069, 1994.

Tnfections acquired by the elderly in long-term care faciliIties are a growing problem. ${ }^{1-3}$ Many of these infections are caused by antibiotic-resistant bacteria. ${ }^{4-12}$ Most studies have focused on infections caused by one particular antibiotic-resistant microorganism and have often been initiated by the occurrence of an outbreak. ${ }^{12-16}$ Many have focused on Gram-negative bacilli resistant to aminoglycosides or cephalosporins. ${ }^{6,15,16}$ Recently, several reports have emphasized the role of antibiotic-resistant Gram-positive cocci, specifically methicillin-resistant Staphylococcus aureus (MRSA) ${ }^{8-14}$ and high level aminoglycoside-resistant enterococci (R-ENT). ${ }^{7}$ Very few studies have prospectively defined the prevalence and incidence of colonization and infection with several different antibiotic-resistant microorganisms over a prolonged period of time. In this 2-year study, we prospectively determined colonization and infection with MRSA, R-ENT, and ceftriaxone- and/or gentamicinresistant Gram-negative bacilli (R-GNB) and evaluated the factors associated with patient acquisition of these organisms.

\section{METHODS}

\section{Facility and Patients}

The Ann Arbor Veterans Affairs Nursing Home Care Unit is a 120-bed long-term care facility attached to a 300 -bed acute care hospital. There are three services in the 
facility: (1) the geriatric evaluation unit to which all patients are admitted from the acute care hospital for initial evaluation and into which a patient would be transferred, if necessary, for closer medical supervision; (2) the rehabilitation service for patients requiring intensive physical, occupational, or speech therapy, or for patients with stable medical conditions finishing a specified course of therapy for some medical condition; and (3) a long-term care service for patients who have no active medical problem but require a supervised living situation. At any given time, the numbers of patients generally are equally distributed among these three services; however, the mean length of stay among the three services varies: for geriatric evaluation it is 3 months, for rehabilitation 8 months, and for long-term care it is 2 years. The three services are not physically separated. The same nursing staff care for patients on all three services, and patients transfer back and forth between services, depending on their condition. On admission, patients are classified by functional status: Group I are ambulatory and require minimal assistance in activities of daily living; group II require moderate assistance in activities of daily living and have bladder incontinence; and group III require assistance for most activities of daily living and have incontinence of bowel, bladder, or both. ${ }^{17}$

\section{Study Design}

The study of MRSA, R-ENT, and R-GNB colonization was part of a 2-year project to assess colonization and infection with antibiotic-resistant bacteria and the effect on patient care costs in a long-term care facility. ${ }^{9}$ Beginning in June 1989 and continuing through May 1991, all patients in the unit were evaluated with regard to demographics, diagnoses, clinical factors, nutritional parameters, and colonization and infection with MRSA, R-ENT, and R-GNB. Cultures were done at admission for each of the microorganisms studied, monthly thereafter, and at the time of transfer back to long-term care after any hospital admissions. The Human Studies Subcommittee and Research Committee of the Ann Arbor Veterans Affairs Medical Center approved the study protocol and felt that verbal informed consent was adequate.

In the first year of the study, June 1989 to May 1990, colonization was assessed as described. Staff caring for the patients were not made aware of culture results. Standard infection control methods (Universal Precautions combined with category specific precautions, such as contact isolation and respiratory precautions, when required) were used. Patients were treated for infections by their physicians in accordance with cultures obtained for clinical reasons through the clinical microbiology laboratory.

In the second year of the study, June 1990 to May 1991, colonization with MRSA, R-ENT, and R-GNB was assessed in the same manner as the first year. However, an intervention was used in an attempt to decrease MRSA colonization. In all MRSA carriers, mupirocin ointment in a polyethylene glycol base was applied to nares alone from June 1990 to December 1990 and to nares and wounds from January 1991 to June 1991 , as described previously. ${ }^{18}$ No specific intervention was attempted to decrease colonization with R-ENT or R-GNB. Other than knowledge about MRSA colonization resulting from participation in the mupirocin trial, staff were not made aware of the results of cultures obtained in this study. Again, patients were treated for infections by their physicians based on cultures obtained for clinical reasons through the clinical microbiology laboratory.

\section{Patient Factors}

On admission, data, including age, sex, functional status using an adapted Katz index of activities of daily living (ADL), ${ }^{17}$ presence of indwelling tubes or catheters, bladder and bowel function, ability to walk, transfer, and eat unattended, diagnosis prompting admission, underlying illnesses, previous hospitalization, length of stay in both acute and long-term care, and geographic location, movement, and roommates within the long-term care facility, were recorded for all patients.

\section{Definitions of Colonization and Infection}

Colonization was defined by one or more positive cultures for MRSA, R-ENT, or R-GNB from any site. Prospective surveillance for the occurrence of infection was carried out over the 24-month period in all patients in the long-term care facility by a nurse clinician trained in infection control. Each infection was discussed by a panel of three physicians and the nurse clinician to determine whether it met criteria for nosocomial infection as defined by the Centers for Disease Control and Prevention. ${ }^{19}$ Data on colonization was not utilized to determine the presence of infection.

\section{Nutritional Measurements}

Serum albumin and blood lymphocyte numbers were measured by standard assays when patients entered the study. Anthropometric measurements were obtained using skin-fold calipers and a plastic tape to measure triceps skin-fold thickness and upper arm circumference. The upper arm muscle area and fat area were calculated as detailed previously. ${ }^{20}$ Results were expressed as percent of expected, using Frischano's age-related norms. ${ }^{21}$

\section{Microbiologic Methods}

Anterior nares, oropharynx, perineum, rectum, and wounds were swabbed with sterile rayon-tipped applicator sticks. The swabs used for culturing the perineum were pre-moistened with the transport media contained in the culturette; the swabs used on the other sites were not. The swabs were immediately placed into Stuart transport media, plated within 2 hours, and the plates incubated at $35^{\circ} \mathrm{C}$.

Colonization with MRSA was assessed in nares, wounds, rectum, and perineum by streaking the swabs onto colistin-nalidixic acid (CNA) agar containing 5\% sheep's blood. Yellow and white colonies that were Gram-positive cocci in clusters on Gram's stain were identified as S. aureus by positive catalase test, positive Accu-Staph latex agglutination test for protein A (Carr-Scarborough Microbiologics, Decatur, GA), and positive tube coagulase test if the AccuStaph test was equivocal. Methicillin resistance was established if growth occurred after incubation for 24 hours at $35^{\circ} \mathrm{C}$ on Mueller-Hinton agar containing $4 \%$ sodium chloride and $6 \mu \mathrm{g} / \mathrm{mL}$ oxacillin. All isolates were phage-typed by standard methods at the Michigan Department of Public Health Laboratory.

Colonization with R-ENT was assessed in wounds, rectum, and perineum by streaking the swabs on CNA plates as noted for MRSA. Colonies producing alpha or no hemolysis were determined to be enterococci by negative catalase 
test, positive bile esculin test, and growth in $6.5 \%$ sodium chloride. High-level gentamicin-resistance was established if growth occurred on trypticase soy agar containing 500 $\mu \mathrm{g} / \mathrm{mL}$ gentamicin. ${ }^{7}$

Colonization with R-GNB was sought in oropharynx, perineum, rectum, and wounds by streaking the swabs on two separate MacConkey agar plates, one containing 40 $\mu \mathrm{g} / \mathrm{mL}$ gentamicin and the other $100 \mu \mathrm{g} / \mathrm{mL}$ ceftriaxone. R-GNB were identified to the species level with use of the API-20E system (Analytab Products, Plainview, NY).

\section{Statistical Methods}

Results were expressed as mean \pm SE. Data from the first year are reported separate from that of the second year in certain categories because of the intervention to clear MRSA carriage in year two. In the second year, the first 7 months reflects the use of mupirocin in the anterior nares of MRSA carriers, and the second 5 months reflects the use of mupirocin in both anterior nares and wounds of MRSA carriers. The chi-square test or one-way analysis of variance was used to compare colonization rates.

For risk factor analysis, initial univariate analysis was done by using chi-square or Fisher's exact test for ordinal variables with two levels, Mann-Whitney test for ordinal variables with greater than two levels, and Student's $t$ test for continuous variables. In order to evaluate the independent effect of each factor shown to be significant for colonization or infection with each organism by univariate analysis, a stepwise logistic regression model was developed using those variables that were significant at $P<0.05$ in the univariate analysis.

\section{RESULTS}

A total of 551 patients were entered into the study. There were 542 men and nine women; the mean age was $64.4 \pm 0.5$ years.

\section{Rates of Colonization}

Colonization at any site was compared for MRSA, R-ENT, and R-GNB in the first year and for the two periods in the second year (Figure 1). While the rate of colonization with R-ENT and R-GNB was relatively constant in all three time periods, MRSA colonization fell significantly after the introduction of mupirocin treatment of both anterior nares and wounds of MRSA carriers from January through June $1991(P=0.0001)$. Except in the third period, colonization rates were higher for both MRSA and R-ENT than for R-GNB.

Considering only the first year of the study before mupirocin use and only those three sites in which all three types of resistant organisms were sought (rectum, perineum, and wounds), differences emerged in the pattern of colonization by each type of organism (Figure 2). MRSA was an uncommon colonizer in perineum and rectum and was most often found colonizing wounds, whereas R-ENT colonized all three sites at about the same frequency. R-GNB colonization was the least common in all three sites, and wounds were colonized more often than perineum and rectum.

Not reflected in Figure 2 are other sites of colonization by MRSA and R-GNB. Other than wounds, the highest colonization rates were in anterior nares for MRSA (15.1 \pm $1 \%$ of patients colonized per month during the first year of the study). Colonization of the oropharynx was determined
Figure 1. Mean ( $\pm S E$ ) monthly rate of colonization with antibiotic-resistant microorganisms for the periods listed. $M R S A=$ methicillin-resistant $S$. aureus; $R-E N T=$ gentamicinresistant enterococcus; $R-G N B=$ gentamicin and/or ceftriaxone-resistant Gram-negative bacilli. Colonization rates fell significantly for MRSA colonization in the period 1/91-6/91 after mupirocin ointment was used in wounds and anterior nares of carriers, $P=0.0001$ by one-way analysis of variance comparing all three time periods.

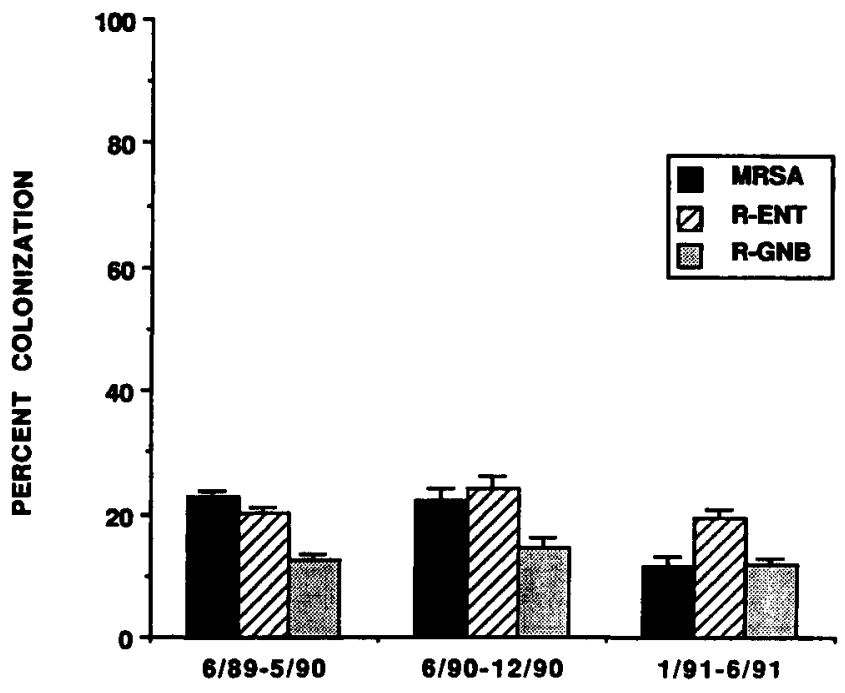

only for R-GNB. Over the 2-year period of the study, $4.3 \pm$ $0.6 \%$ of patients each month had R-GNB cultured from the oropharynx.

The most common species of R-GNB colonizing patients were Acinetobacter species $(16.5 \%$ of colonized patients), Pseudomonas species (14.5\%), Pseudomonas aeruginosa (11.9\%), Klebsiella pneumoniae (10\%), Escherichia coli $(8.7 \%)$, and Enterobacter cloacae $(8.7 \%)$.

Figure 2. Mean ( $\pm S E$ ) monthly rate of colonization by antibiotic-resistant bacteria at various sites during the first year of the study (6/89-5/90). MRSA = methicillin-resistant S. aureus; $R-E N T=$ gentamicin-resistant enterococcus; $R-G N B=$ gentamicin and/or ceftriaxone-resistant Gram-negative bacilli. Wound data are expressed as the percent of patients who had wounds that could be cultured.

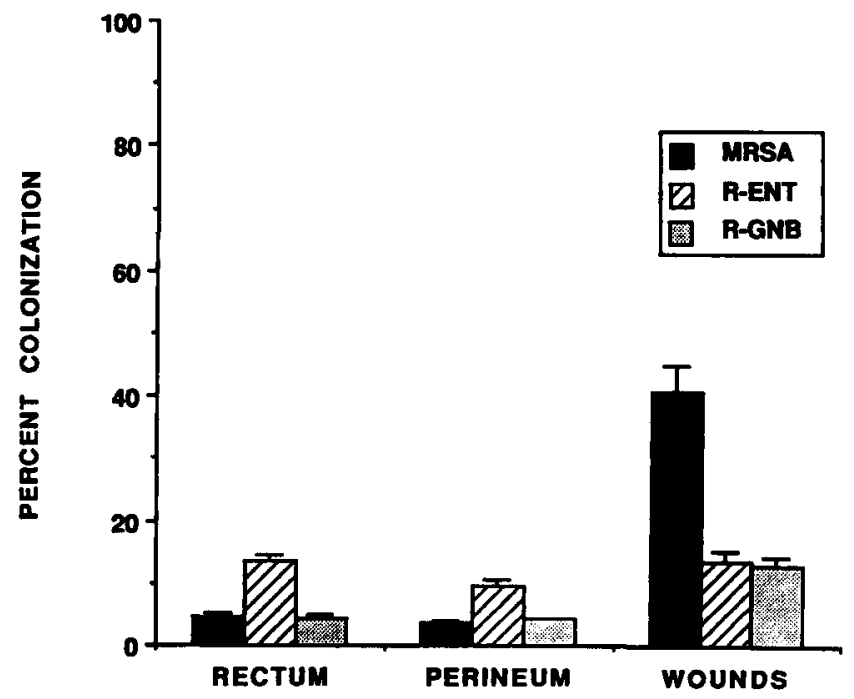


Risk Factors for Colonization with Antibiotic-Resistant Bacteria

Evaluation of risk factors for MRSA colonization was carried out for year 1 when no measures had been taken to change colonization patterns. Twelve variables were found to be associated with MRSA colonization at any site (Table 1); however, only two of these variables - presence of wounds and decubitus ulcers - were independently associated with MRSA colonization by logistic regression analysis (Table 2).

Since there were no changes noted with colonization with R-ENT as a result of the intervention to decrease MRSA carriage, data from both years of the study were combined in the analysis of risk factors associated with colonization. Univariate analysis showed 12 factors associated with colonization at any site with R-ENT; many of these were markers for poor functional status (Table 3). Logistic regression revealed five independent variables associated with R-ENT colonization (Table 2). Colonization at any site with R-GNB was associated with 10 different factors (Table 4), but stepwise logistic regression analysis revealed only five independent variables associated with colonization (Table 2).

\section{Rates of Infection}

Because of the intervention to decrease MRSA colonization and, presumably, infections, data are divided into infections in the first year (6/89-5/90) and in the two periods in the second year. Overall, there were 326 infections caused by antibiotic-resistant and antibiotic-susceptible bacteria acquired in the long-term care facility - 181 in year one, 64 in the period June through December 1990, and 81 in the period January through June 1991 (a total of 145 in year 2).

Table 1. Univariate analysis of risk factors associated with colonization by methicillin-resistant Staphylococcus aureus prior to the use of mupirocin ointment

\begin{tabular}{lccc}
\hline & \multicolumn{2}{c}{ Percent of Patients } \\
\cline { 2 - 3 } \multicolumn{1}{c}{ Factor } & Colonized & Not \\
Colonized & $P$ Value \\
\cline { 2 - 3 } Underlying conditions & & & \\
Presence of wounds & 40.3 & 19.0 & 0.0001 \\
Decubitus ulcers & 18.0 & 7.6 & 0.005 \\
Prior pneumonia & 20.1 & 9.9 & 0.009 \\
Anemia & 37.0 & 23.2 & 0.009 \\
Chronic renal failure & 19.4 & 9.8 & 0.01 \\
Peptic ulcer D. & 24.8 & 14.3 & 0.01 \\
Chronic arthritis & 48.4 & 35.3 & 0.02 \\
Coronary artery D. & 39.5 & 28.4 & 0.04 \\
Katz Functional Scale & & & \\
I & 17.8 & 31.4 & 0.0003 \\
II & 48.1 & 48.8 & \\
III & 34.1 & 19.8 & \\
Nutritional parameters & & & \\
$\quad(\bar{\chi} \pm$ SD) & & & \\
Serum albumin & $3.7 \pm 0.6$ & $4.0 \pm 0.6$ & 0.004 \\
Lymphocyte number & $1689 \pm 736$ & $1914 \pm 711$ & 0.005 \\
Lymphocyte percent & $22.2 \pm 8.6$ & $24.2 \pm 8.9$ & 0.04 \\
\hline
\end{tabular}

Table 2. Factors shown by stepwise logistic regression to be independently associated with colonization by antibioticresistant bacteria

\begin{tabular}{llcc}
\hline \multicolumn{1}{c}{ Factor } & $\begin{array}{c}\text { Odds } \\
\text { Ratio }\end{array}$ & $\begin{array}{c}95 \% \text { Confidence } \\
\text { Interval }\end{array}$ & $\begin{array}{c}P \\
\text { Value }\end{array}$ \\
\hline $\begin{array}{llll}\text { MRSA } \\
\quad \text { Presence of wounds }\end{array}$ & 3.1 & $1.8-5.4$ & 0.0001 \\
$\quad \begin{array}{l}\text { Decubitus ulcer } \\
\text { R-ENT }\end{array}$ & 2.7 & $1.1-6.5$ & 0.027 \\
$\quad$ Presence of wounds & 2.6 & $1.7-3.9$ & 0.0001 \\
$\quad$ Renal failure & 2.4 & $1.4-4.3$ & 0.002 \\
$\quad$ Intermittent cath. & 2.4 & $1.3-4.3$ & 0.004 \\
$\quad$ Katz functional scale & 1.8 & $1.3-2.5$ & 0.0001 \\
$\quad$ Serum albumin & 1.6 & $1.1-2.2$ & 0.015 \\
R-GNB & & & \\
$\quad$ Intermittent cath. & 3.9 & $2.2-6.9$ & 0.0001 \\
$\quad$ Inflammatory bowel D. & 3.4 & $1.2-9.9$ & 0.022 \\
$\quad$ Chronic renal D. & 2.7 & $1.1-6.8$ & 0.035 \\
$\quad$ Presence of wounds & 1.9 & $1.2-3.0$ & 0.008 \\
$\quad$ Prior pneumonia & 1.9 & $1.0-3.6$ & 0.038 \\
\hline
\end{tabular}

MRSA = methicillin-resistant $S$. aureus; R-ENT = gentamicin-resistant enterococcus; R-GNB = gentamicin- and/or ceftriaxone-resistant Gram negative bacilli; cath. $=$ urethral catheterization.

The predominant infections were urinary tract infections (42.9\% of all infections over 2 years) and skin and soft tissue infections (31.9\% of all infections over 2 years) (Table 5).

In $102(31.3 \%)$ of the 326 infections, most of which were lower respiratory tract infections and skin and soft tissue infections, specifically cellulitis, the organism causing infection was not known. Of 224 infections in which the

Table 3. Univariate analysis of factors associated with colonization by gentamicin-resistant enterococci

\begin{tabular}{|c|c|c|c|}
\hline \multirow[b]{2}{*}{ Factor } & \multicolumn{2}{|c|}{ Percent of Patients } & \multirow[b]{2}{*}{$\begin{array}{c}P \\
\text { Value }\end{array}$} \\
\hline & Colonized & $\begin{array}{c}\text { Not } \\
\text { Colonized }\end{array}$ & \\
\hline \multicolumn{4}{|l|}{ Underlying conditions } \\
\hline Presence of wounds & 46.0 & 25.4 & 0.0001 \\
\hline Chronic renal failure & 20.3 & 8.7 & 0.0004 \\
\hline Recurrent UTIs & 19.9 & 9.5 & 0.0009 \\
\hline Neurogenic bladder & 38.5 & 9.5 & 0.003 \\
\hline Prior pneumonia & 17.2 & 9.8 & 0.008 \\
\hline Decubitus ulcers & 21.7 & 14.5 & 0.02 \\
\hline Diabetes mellitus & 36.6 & 26.6 & 0.02 \\
\hline Peripheral vascular D. & 36.5 & 27.3 & 0.02 \\
\hline \multicolumn{4}{|l|}{ Devices, Procedures } \\
\hline Intermittent cath. & 23.5 & 10.1 & 0.0001 \\
\hline Gastrostomy tube & 5.0 & 1.4 & 0.02 \\
\hline \multicolumn{4}{|l|}{ Katz Functional Scale } \\
\hline 1 & 14.6 & 38.3 & 0.0001 \\
\hline II & 51.5 & 44.5 & \\
\hline III & 33.8 & 17.1 & \\
\hline \multicolumn{4}{|l|}{ Nutritional parameters } \\
\hline Serum albumin ( $\bar{\chi} \pm$ S.D. $)$ & $3.6 \pm 0.6$ & $3.8 \pm 0.6$ & 0.0001 \\
\hline
\end{tabular}

cath. $=$ urethral catheterization. 
Table 4. Univariate analysis of factors associated with colonization with gentamicin-and/or ceftriaxone-resistant Gram-negative bacilli

\begin{tabular}{lrrr}
\hline & \multicolumn{2}{c}{ Percent of Patients } \\
\cline { 2 - 3 } \multicolumn{1}{c}{ Factor } & \multicolumn{3}{c}{$\begin{array}{c}\text { Not } \\
\text { Colonized }\end{array}$} \\
\cline { 2 - 3 } & Colonized & Value \\
\hline Underlying conditions & & & \\
Recurrent UTIs & 20.5 & 10.8 & 0.007 \\
Presence of wounds & 44.2 & 31.6 & 0.01 \\
Inflammatory bowel D. & 7.0 & 2.3 & 0.02 \\
Prior pneumonia & 17.8 & 10.7 & 0.03 \\
Chronic renal D. & 7.8 & 3.2 & 0.03 \\
Osteoarthritis & 19.4 & 12.6 & 0.04 \\
Devices, Procedures & & & \\
Intermittent cath. & 27.9 & 11.0 & 0.0001 \\
Gastrostomy tube & 6.2 & 1.7 & 0.01 \\
Katz Functional Scale & & & \\
I & 23.6 & 30.5 & 0.04 \\
II & 47.2 & 47.9 & \\
III & 29.1 & 21.7 & \\
Nutritional parameters & & & \\
Serum albumin $(\bar{\chi} \pm$ S.D.) & $3.7 \pm 0.6$ & $3.8 \pm 0.6$ & 0.04 \\
\hline
\end{tabular}

cath. = urethral catheterization.

organism was documented, $111(49.6 \%)$ were due to the antibiotic-resistant bacteria, MRSA, R-ENT, or R-GNB, and $113(50.4 \%)$ were due to susceptible staphylococci, streptococci, Gram-negative bacilli, and other organisms.

\section{Infections Caused by Gram-Negative Bacilli}

The most common infecting organisms were Gramnegative bacilli, but the majority were not antibioticresistant (Table 6). Of a total of 153 infections caused by Gram-negative bacilli, $69(45.1 \%)$ were gentamicin and/or ceftriaxone resistant. The Gram-negative bacilli causing most infections were $P$. aeruginosa $(20.9 \%$ of GNB infections), P. mirabilis (18.9\%), K. pneumoniae (17\%), and $E$. coli $(15.7 \%)$. Acinetobacter species and non-aeruginosa Pseudomonas species, although the most common coloniz-

Table 5. Site of infections caused by both antibiotic-susceptible and antibiotic-resistant bacteria acquired in a long-term care facility over a period of 2 years

\begin{tabular}{lccc}
\hline & \multicolumn{3}{c}{$\begin{array}{c}\text { Number (\%) of Infections } \\
\text { in Each Period* }\end{array}$} \\
\cline { 2 - 4 } \multicolumn{1}{c}{$\begin{array}{c}\text { Types of } \\
\text { Infections }\end{array}$} & $6 / 89-5 / 90$ & $6 / 90-12 / 90$ & $1 / 91-6 / 91$ \\
\hline Urinary tract & $78(43.1)$ & $25(39.9)$ & $37(45.7)$ \\
Skin/soft tissue & $59(32.6)$ & $17(27.0)$ & $28(34.5)$ \\
Lower respiratory & $29(16.0)$ & $14(22.2)$ & $8(9.9)$ \\
Bone/joint & $8(4.4)$ & $1(1.5)$ & $3(3.7)$ \\
Bacteremia & $4(2.2)$ & $5(7.9)$ & $2(2.5)$ \\
Other & $3(1.7)$ & $1(1.5)$ & $3(3.7)$ \\
Total & 181 & 64 & 81 \\
\hline
\end{tabular}

* In periods $6 / 90-12 / 90$ and $1 / 91-6 / 91$, attempts were made to eradicate MRSA colonization in all patients in the facility.
Table 6. Bacterial infections acquired in a long-term care facility

Number (\%) of Infections in Each Period

\begin{tabular}{lccc}
\cline { 2 - 4 } Organism & $6 / 89-5 / 90$ & $6 / 90-12 / 90$ & $1 / 91-6 / 91$ \\
\hline Gram-positive cocci & $37(20.5)$ & $12(18.8)$ & $22(27.1)$ \\
MRSA & $9(5.0)$ & $3(4.7)$ & $3(3.7)$ \\
MSSA & $5(2.8)$ & 0 & $4(4.9)$ \\
R-ENT & $15(8.3)$ & $7(10.9)$ & $5(6.2)$ \\
S-ENT & $5(2.8)$ & $1(1.6)$ & $7(8.6)$ \\
Other (S. epidermidis, & $3(1.6)$ & $1(1.6)$ & $3(3.7)$ \\
S. pneumoniae) & & & \\
Gram-negative bacilli & $90(49.7)$ & $29(45.3)$ & $34(42.0)$ \\
R-GNB & $41(22.6)$ & $15(23.4)$ & $13(16.0)$ \\
S-GNB & $45(24.9)$ & $14(21.9)$ & $19(23.5)$ \\
Other (Haemophilus) & $4(2.2)$ & 0 & $2(2.5)$ \\
Unknown organism & $54(29.8)$ & $23(35.9)$ & $25(30.9)$ \\
Total & 181 & 64 & 81
\end{tabular}

MRSA = methicillin-resistant $S$. aureus; MSSA = methicillin-susceptible $S$. aureus; R-ENT = gentamicin-resistant enterococcus; S-ENT = enterococcus lacking high-level gentamicin resistance; R-GNB = gentamicin- and/or ceftriaxone-resistant Gram bacilli; S-GNB = gentamicin and ceftriaxone susceptible gram negative bacilli.

ers, rarely caused infection. As expected, susceptibility to gentamicin and ceftriaxone varied among species of Gramnegative bacilli. Of $32 P$. aeruginosa infections, 27 (84.4\%) were caused by resistant strains. $P$. mirabilis, the second most common infecting organism, was resistant in $58.6 \%$ of the episodes of infections. In contrast, most $E$. coli and $K$. pneumoniae infections were due to susceptible strains (83.3\% and $88.5 \%$, respectively). Most often Gramnegative bacilli caused urinary tract infections; much less commonly they caused skin and soft tissue infections and lower respiratory tract infections. $P$. aeruginosa was the most common Gram-negative bacillus causing skin and soft tissue infections, as well as pneumonia.

\section{Infections Caused by Gram-Positive Cocci}

Infections caused by Gram-positive cocci were less common than those caused by Gram-negative bacilli, but were more likely to be antibiotic-resistant (Table 6). Among 24 culture-proved staphylococcal infections, MRSA were more common than MSSA (62.5\% vs $37.5 \%)$. However, this is undoubtedly an under-representation of the number of MSSA infections. Many infections for which no pathogen was isolated were skin and soft tissue infections, usually cellulitis. Most were treated with antibiotics, which would not be effective for MRSA, improved on therapy, and were likely the result of MSSA or streptococci. Of the culturedocumented staphylococcal infections, all of the nine MSSA infections were skin and soft tissue infections or osteomyelitis, and 13 of 15 MRSA infections were skin and soft tissue infections or osteomyelitis, including two patients with bacteremia.

Enterococcal infections were more often caused by gentamicin-resistant strains than susceptible strains $(67.5 \%$ vs $32.5 \%$ ). Most enterococcal infections (75\%) were urinary tract infections, and $73.3 \%$ of the urinary tract infections were caused by gentamicin-resistant strains. The remainder of the enterococcal infections were skin and soft tissue 
infections, usually wounds, and equal numbers were due to resistant and susceptible strains of enterococci.

\section{Risk Factors for Infection with Antibiotic-Resistant Bacteria}

Stepwise logistic regression showed several factors to be significant for predicting infection with MRSA and R-GNB (Table 7). MRSA infection was associated with the presence of diabetes mellitus and peripheral vascular disease, and R-GNB infection was associated with the use of either indwelling Foley catheters or intermittent urethral catheterization. R-ENT infection was not associated with any one specific risk factor.

\section{DISCUSSION}

This study provides insight into the role antibioticresistant organisms play in colonization and infection in the long-term care setting. Having not been prompted by an outbreak, it differs from other studies, which have focused on the spread of one antibiotic-resistant organism within a facility. ${ }^{12,13,16}$ Although similar to other prevalence surveys of infections in long-term care, ${ }^{1-3,22-26}$ it differs in that it specifically assesses the contribution of antibiotic-resistant organisms to the overall problem of infections in long-term care.

Colonization with antibiotic-resistant organisms, especially Gram-positive cocci, was common. The rate of colonization with MRSA was similar to that noted by others studying similar facilities, ${ }^{10,11}$ but clearly much higher than that noted in other non-veterans facilities. ${ }^{22}$ Although we previously noticed an association of MRSA colonization with poor functional status, ${ }^{9}$ further analysis using stepwise logistic regression methods showed the only independent risk factors to be the presence of wounds and decubitus ulcers. However, in the case of infections with MRSA, wounds and decubitus ulcers were not significant risk factors; rather, the presence of diabetes mellitus and peripheral vascular disease showed strong associations with MRSA infection. Thus, colonization of wounds with MRSA was common, but most patients did not develop infection unless other factors associated with poor vascular supply were present as well.

Although there were only nine MRSA infections before the eradication efforts with mupirocin and six during the eradication phase, these infections often were severe. For example, two patients had bacteremia, five had osteomyeli-

Table 7. Factors shown by stepwise logistic regression to be associated with infections caused by antibiotic-resistant bacteria

\begin{tabular}{lrcc}
\hline \multicolumn{1}{c}{ Factor } & $\begin{array}{c}\text { Odds } \\
\text { Ratio }\end{array}$ & $\begin{array}{c}\text { 95\% Confidence } \\
\text { Interval }\end{array}$ & $\begin{array}{c}P \\
\text { Value }\end{array}$ \\
\hline $\begin{array}{l}\text { MRSA } \\
\quad \text { Diabetes mellitus }\end{array}$ & 5.1 & $2.1-18.6$ & 0.001 \\
$\quad \begin{array}{l}\text { Peripheral vascular D. } \\
\text { R-GNB }\end{array}$ & 4.3 & $1.3-14.3$ & 0.019 \\
$\quad$ Intermittent cath. & 5.0 & $2.2-11.3$ & 0.0001 \\
$\quad$ Foley catheter & 11.9 & $1.2-119$ & 0.03 \\
\hline
\end{tabular}

MRSA = methicillin-resistant $S$. aureus; $\mathrm{R}-\mathrm{GNB}=$ gentamicin and/or ceftriaxone resistant Gram negative bacilli; cath. = urethral catheterization. tis, and 10 of 15 episodes required hospitalization for therapy with intravenous vancomycin. ${ }^{9,18}$

Our efforts to eradicate MRSA with mupirocin did not decrease the number of infections significantly, ${ }^{18}$ probably because we had a low rate of infection to begin with, but did lead to the selection of MRSA strains carrying a plasmid for mupirocin resistance. ${ }^{27}$ Perhaps decolonization should be specifically aimed at those at highest risk of developing infection, ie, those patients with diabetes mellitus and peripheral vascular disease. If targeted decolonization were to be performed, as is done in hemodialysis patients, who are at increased risk of $S$. aureus infections, ${ }^{28}, 29$ then perhaps the development of mupirocin resistance would be less likely than that seen when mupirocin is used chronically and in a widespread fashion. ${ }^{18,27,30}$

Increasing antibiotic resistance has become a major problem among enterococci. High-level resistance to gentamicin as a marker for resistance to all aminoglycosides first emerged as a major problem in the 1980 s and has since spread widely. ${ }^{7,31,32}$ Even more worrisome is the recent spread of resistance to penicillins and to vancomycin. ${ }^{33,34}$ Neither of these resistance pattterns were sought in our study since they had not yet appeared in our medical center at the time the study was begun in June 1989.

Colonization with R-ENT was associated with many underlying risk factors, many of which were found with MRSA also. Colonization patterns with R-ENT were similar to those for MRSA in regard to acquisition in the facility. ${ }^{9}$ However, persistence of colonization was very different from that noted with MRSA; patients were more likely to have only transient or intermittent R-ENT colonization and frequently had new strains appear over time. ${ }^{35}$ Only five factors were independently associated with R-ENT colonization, and no one factor emerged as independently associated with R-ENT infection.

R-ENT strains were more than twice as likely as those strains lacking high-level gentamicin resistance to cause infection; most infections with R-ENT were urinary tract infections. These infections responded well to therapy with amoxicillin and did not require aminoglycosides for synergistic killing. ${ }^{32,36}$ Thus, in comparison with MRSA, R-ENT infections were more common, but were not as severe, did not require hospitalization, and were easily treated with oral therapy.

Although colonization with R-GNB was lower in all sites than R-ENT or MRSA, those organisms were more likely to cause infection. Other studies of infections in long-term care facilities have noted Gram-negative bacillary infections as a major problem. ${ }^{22,24,25}$ Those studies that also assessed antibiotic resistance found that resistance is common in Gram-negative bacilli causing infections in longterm care facilities, ${ }^{4,16,24,37,38}$ and also have noted that transmission can occur from patient-to-patient, probably via hands of health care workers in this setting. ${ }^{6,15}$

Although several underlying risk factors were associated with R-GNB colonization, infection was associated only with intermittent urethral catheterization or chronic indwelling Foley catheters. In many studies, indwelling catheters have been associated with a markedly increased risk of colonization, infection, and selection of antibioticresistant Gram-negative bacilli. 1, 2, 25, 37 In our facility, we have few patients with indwelling urinary catheters and many more in whom intermittent catheterization is per- 
formed. Although infection rates tend to be lower with intermittent catheterization than with indwelling catheters, ${ }^{39}$ this practice was significantly associated with infection with R-GNB.

Overall, urinary tract infections were the most common infections, as noted by other studies in long-term care. ${ }^{1,22-25}$ Skin and soft tissue infections were also commonly seen. However, respiratory infections accounted for only $16 \%$ of the infections acquired in the facility, a figure lower than that noted by others. ${ }^{24,26}$ Bacteremias were uncommon, occurring in only 11 patients over the course of 2 years, and were mostly related to urinary tract infections.

We do not know whether these data are broadly applicable to other long-term care facilities. Except for the work of Scheckler, ${ }^{22}$ most studies on the role of antibioticresistant organisms in the long-term care setting have been carried out in Veterans Affairs facilities ${ }^{6,7,9-11,15,18}$ or have been done only in patients from long-term care facilities transferred to an acute care hospital for an acute illness. ${ }^{4,8} \mathrm{~A}$ decade ago, in eight rural Wisconsin nursing homes in which $75 \%$ of the residents were women, Scheckler found no MRSA and only three gentamicin-resistant Gram-negative bacilli. $^{22}$ Whether the rate of antibiotic resistance in such rural areas has remained this low in the last decade is not known.

Veterans Affairs long-term care facilities tend to report higher rates of colonization with antibiotic-resistant organisms. Perhaps this is related to the frequent movement of patients to the acute care facility, which is often attached to the nursing home care unit. For example, we noted that $20 \%$ of patients with MRSA colonization brought the organism in from the acute care hospital and only $10 \%$ acquired MRSA in the long-term care facility. ${ }^{9}$ Movement between hospitals and long-term care units by physicians, especially house staff, who care for patients in many Veterans Affairs longterm care facilities, may contribute to the spread of some resistant organisms. ${ }^{40,41}$

Perhaps more important than the above points are patient factors. Even within different Veterans Affairs facilities, there are differences in the types of patients cared for; underlying diseases, acuity of illness, and functioning of the unit as an intermediate care unit, a rehabilitation unit, or a long-term care unit may explain differences noted in rates of infection with antibiotic-resistant organisms in different veterans facilities. ${ }^{9-11}$ In general, it appears that the more ill the patients served and the more skilled care required, the greater the infection rates with antibiotic-resistant organisms. ${ }^{9,10}$

Similar studies should be repeated in other types of long-term care facilities, such as community-based and private facilities, and in different geographic areas of the country to assess the importance of infection with antibioticresistant organisms. Only then can effective approaches be formulated to deal with the problem. For now, one approach might be to modify, when feasible, those factors that appear to be associated with the highest risk of infection. For example, decreasing the use of indwelling urethral catheters and using careful techniques for intermittent catheterization are measures that could be undertaken. Preventing skin breakdown with subsequent development of decubitus ulcers should be a priority. Perhaps specific decolonization efforts for certain organisms, such as MRSA, might be undertaken, but only in high-risk patients.

\section{REFERENCES}

1. Franson TR, Duthie EH, Cooper JE et al. Prevalence survey of infections and their predisposing factors at a hospital-based nursing home care unit. J Am Geriatr Soc 1986;34:95-100.

2. Garibaldi RA, Brodine S, Matsumiya S. Infections among patients in nursing homes: Policies, prevalence, and problems. N Engl J Med 1981;305:731-5.

3. Magnussen $\mathrm{MH}$, Robb SS. Nosocomial infections in a long term care facility. Am J Infect Control 1980;8:12-17.

4. Gaynes RP, Weinstein RA, Chamberlin W et al Antibiotic-resistant flora in nursing home patients admitted to the hospital. Arch Intern Med 1985;145:1864-7.

5. John JE, Ribner BS. Antibiotic resistance in long-term care facilities. Infect Control Hosp Epidemiol 1991;12:245-50.

6. Wingard E, Shlaes JH, Mortimer EA et al. Colonization and crosscolonization of nursing home patients with trimethoprim-resistant Gramnegative bacilli. Clin Infect Dis 1993;16:75-81.

7. Zervos MJ, Terpenning MS, Schaberg DR et al. High level aminoglycosideresistant enterococci: Colonization of nursing home and acute care hospital patients. Arch Intern Med 1987;147:1591-4.

8. Hsu CCS, Macaluso CP, Special L et al. High rate of methicillin-resistance of Staphylococcus aureus isolated from hospitalized nursing home patients. Arch Intern Med 1988;148:569-70.

9. Bradley SF, Terpenning MS, Ramsey MA et al. Methicillin-resistant Staphylococcus aureus: Colonization and infection in a long-term care facility. Ann Intern Med 1991;115:417-22.

10. Muder RR, Brennen $C$, Wagener $M M$ et al. Methicillin-resistant staphylococcal colonization infection in a long-term care facility. Ann Intern Med 1991;114:107-12.

11. Strausbaugh LJ, Jacobson C, Sewell DL et al. Methicillin-resistant Staphylococcus aureus in extended-care facilities. Experience in a Veterans Affairs nursing home and a review of the literature. Infect Control Hosp Epidemiol 1991;12:36-45.

12. O'Toole RD, Drew WL, Dahlgren BV et al. An outbreak of methicillinresistant Staphylococcus aureus infection. Observations in hospital and nursing home. JAMA 1970;213:257-63.

13. Storch GA, Radcliff JL, Meyer PL et al. Methicillin-resistant Staphylococcus aureus in a nursing home. Infect Control 1987;8:24-9.

14. Boyce JM. Methicillin resistant Staphylococcus aureus in nursing homes: Putting the problem in perspective. Infect Control Hosp Epidemiol 1991;12:413-15.

15. Shlaes DM, Lehman MH, Currie-McCumber CA et al. Prevalence of colonization with antibiotic resistant Gram-negative bacilli in a nursing home care unit: The importance of cross colonization as documented by plasmid analysis. Infect Control 1986;7:538-45.

16. Rice LB, Willey SH, Papanicolaou GH et al. Outbreak of ceftazidime resistance caused by extended-spectrum $\beta$-lactamases at a Massachusetts chronic-care facility. Antimicrob Agents Chemother 1990;34:2193-9.

17. Katz S, Ford AB, Moskowitz RW et al. Studies of illness in the aged. The index of ADL: A standardized measure of biological and psychosocial function. JAMA 1963;185:914-19.

18. Kauffman CA, Terpenning MS, Zarins LT et al. Attempts to eradicate methicillin-resistant Staphylococcus aureus from a long-term care facility with the use of mupirocin ointment. Am J Med 1993;54:371-8.

19. Garner JS, Jarvis WR, Emori TG et al. CDC definitions for nosocomial infections. Am J Infect Control 1988;16:128-40.

20. Bradley SF, Vibhagool A, Fabrick $S$ et al. Monokine production by malnourished nursing home patients. Gerontology 1990;36:165-70.

21. Frischano AR. New norms of upper limb fat and muscle areas for assess ment of nutritional status. Am J Clin Nutr 1981;34:2540-5.

22. Scheckler WE, Peterson PJ. Infections and infection control among residents of eight rural Wisconsin nursing homes. Arch Intern Med 1986;146:1981-4.

23. Steinmiller AM, Ross SS, Muder RR. Prevalence of nosocomial infections in long-term care Veterans Administration medical centers. Am J Infect Control 1991;19:143-6.

24. Farber BF, Brennen C, Puntereri AJ et al. A prospective study of nosocomial infections in a chronic care facility. J Am Geriatr Soc 1984;32:499502.

25. Standfast SJ, Michelsen $P B$, Baitch AL et al. A prevalence survey of infections in a combined acute and long-term care hospital. Infect Control 1984;5:177-84

26. Nicolle LE, McIntyre $M$, Zacharias $H$ et al. Twelve-month surveillance of infections in institutionalized elderly men. J Am Geriatr Soc 1984;32:513 19. 
27. Janssen DA, Zarins LT, Schaberg DR et al. Detection and characterization of mupirocin resistance in Staphylococcus aureus. Antimicrob Agents Chemother 1993;37:2003-2006.

28. Yu VL, Goetz A, Wagener $M$ et al. Staphylococcus aureus nasal carriage and infections in patients on hemodialysis: Efficacy of antibiotic prophylaxis. N Engl J Med 1986;315:91-6.

29. Boelaert JR, deSmedt RA, deBaere YA et al. The influence of calcium mupirocin nasal ointment on the incidence of Staphylococcus aureus infections in hemodialysis patients. Nephrol Dial Transplant 1989;4:278-81.

30. Bradley SF. Effectiveness of mupirocin in the control of methicillin-resistant Staphylococcus aureus. Infect Med 1993;10:23-31.

31. Zervos MJ, Kauffman CA, Therasse PM et al. Epidemiology of nosocomial infection caused by gentamicin resistant Streptococcus faecalis. Ann Intern Med 1987;106:687-91.

32. Moellering RC Jr. Emergence of enterococcus as a significant pathogen. Clin Infect Dis 1992;14:1173-8.

33. Boyce JM, Opal SM, Potter-Bynoe G et al. Emergence and nosocomial transmission of ampicillin-resistant enterococci. Antimicrob Agents Chemother 1992;36:1032-9.

34. Livornese LL, Dias $S$, Samel C et al. Hospital-acquired infection with vancomycin-resistant Enterococcus faecium transmitted by electronic thermometers. Ann Intern Med 1992;117:112-16.

35. Chenoweth CE, Bradley SF, Terpenning MS et al. Colonization and trans- mission of highly gentamicin-resistant enterococci in a long-term care facility. Infect Control Hosp Epidemiol, in press.

36. Terpenning MS, Zervos MJ, Schaberg DR et al. Enterococcal infections. An increasing problem in the elderly. Infect Control Hosp Epidemiol 1988;9:457-61.

37. Bjork DT, Pelletier LL, Tight RR. Urinary tract infection with antibiotic resistant organisms in catheterized nursing home patients. Infect Control 1984;5:173-6.

38. Muder RR, Brennen C, Goetz AM et al. Association with prior fluoroquinolone therapy of widespread ciprofloxacin resistance among Gramnegative isolates in a Veterans Affairs medical center. Antimicrob Agents Chemother 1991;35:256-8.

39. Terpenning MS, Allada R, Kauffman CA. Intermittent urethral catheterization in the elderly. J Am Geriatr Soc 1989;37:411-16.

40. Reboli AC, John JF Jr, Platt CG et al. Methicillin-resistant Staphylococcus aureus outbreak at a Veterans Affairs medical center. Importance of carriage of the organism by hospital personnel. Infect Control Hosp Epidemiol 1990;11:343-50.

41. Haley RW, Hightower AW, Khabbaz RF et al. The emergence of methicillin-resistant Staphylococcus aureus infections in United States hospitals: Possible role of the house staff-patient transfer circuit. Ann Intern Med 1982;97:297-308. 